\title{
Clinical Results of Radiotherapy That Targeted for Tumor Thrombi from Hepatocellular Carcinoma: A Multicenter Retrospective Study
}

\author{
Takuya Nagano ${ }^{1,}$, , Akihiko Hoshi ${ }^{2}$, Masayuki Kurosaki ${ }^{3}$, Kazuma Sasamura ${ }^{4}$, Kaoru Tsuchiya ${ }^{3}$, \\ Kazuma Toda ${ }^{1}$, Hirofumi Kuwabara ${ }^{2}$, Meika Namba ${ }^{2}$, Daigoro Matsubara ${ }^{5}$, Sayako Oota ${ }^{5}$, \\ Yasuo Yoshioka ${ }^{4}$, Ryoichi Yoshimura ${ }^{1}$, Namiki Izumi ${ }^{3}$ \\ ${ }^{1}$ Department of Radiation Therapeutics and Oncology, Tokyo Medical and Dental University, Bunkyo-ku, Tokyo, Japan \\ ${ }^{2}$ Department of Radiology, Musashino Red Cross Hospital, Musashino-shi, Tokyo, Japan \\ ${ }^{3}$ Department of Gastroenterology and Hepatology, Musashino Red Cross Hospital, Musashino-shi, Tokyo, Japan \\ ${ }^{4}$ Department of Radiation Oncology, Cancer Institute Hospital of Japanese Foundation for Cancer Research, Koto-ku, Tokyo, Japan \\ ${ }^{5}$ Department of Radiology, Asahi General Hospital, Asahi-shi, Chiba, Japan
}

Email address:

ngnmrad@tmd.ac.jp (T. Nagano)

${ }^{*}$ Corresponding author

\section{To cite this article:}

Takuya Nagano, Akihiko Hoshi, Masayuki Kurosaki, Kazuma Sasamura, Kaoru Tsuchiya, Kazuma Toda, Hirofumi Kuwabara, Meika Namba, Daigoro Matsubara, Sayako Oota, Yasuo Yoshioka, Ryoichi Yoshimura, Namiki Izumi. Clinical Results of Radiotherapy That Targeted for Tumor Thrombi from Hepatocellular Carcinoma: A Multicenter Retrospective Study. International Journal of Clinical Oncology and Cancer Research. Vol. 5, No. 3, 2020, pp. 65-77. doi: 10.11648/j.ijcocr.20200503.13

Received: September 16, 2020; Accepted: September 27, 2020; Published: October 7, 2020

\begin{abstract}
Background: The aim of this multicenter study was to evaluate the outcome of radiotherapy (RT) that targeted for tumor thrombi (TT) from hepatocellular carcinoma (HCC), including the portal vein, hepatic vein, inferior vena cava, and bile duct TT. Methods: Patients who received RT for the treatment of TT between 2005 and 2020 were retrospectively reviewed. We compared patient characteristics, overall survival (OS), the combined chemotherapy regimen, and objective response rates (ORRs) between the treatment modalities and analyzed cumulative incidence formula (CIF) for the deterioration in the Child-Pugh class and the progression of intrahepatic tumors. Results: We evaluated 64 patients, 39 of whom received combined chemotherapy with RT. Multivariate analysis showed that the Child-Pugh class, primary tumor size and the response of TT were significant prognostic factors for OS and the total equivalent dose in 2 Gy fractions (EQD2) of more than 48.75 Gy significantly contributed to ORRs $(p=0.04)$. In the multivariate analysis of CIF, only acute liver damage was the significant factor for the deterioration in the Child-Pugh class $(\mathrm{p}=0.01)$ and the length of TT was significant for the progression of intrahepatic tumors $(\mathrm{p}=0.03)$. Conclusion: High doses should be delivered to TT, but long tumor thrombi are difficult to control. Tumor thrombus length is more important in predicting intrahepatic progression than the location of the tumor thrombus.
\end{abstract}

Keywords: Combined Chemotherapy, Radiation Therapy, Hepatocellular Carcinoma, Macroscopic Vascular Invasion, Tumor Thrombosis

\section{Introduction}

Patients with advanced hepatocellular carcinoma (HCC) often present with the invasion of the hepatic vasculature and bile ducts. They cause extensive intrahepatic dissemination of the tumor. Moreover, portal vein tumor thrombosis (PVTT) decreases the blood supply to the normal liver, and finally causes portal hypertension, ascites, hepatic encephalopathy, and deteriorating liver function [1]. Hepatic vein tumor thrombosis (HVTT) and inferior vena cava tumor thrombosis (IVCTT) may flow into the heart and lung, leading to pulmonary embolism and lung metastasis [2]. Therefore, these conditions can be life-threatening, and their prognosis remains very poor.

Molecular targeted therapy using sorafenib continues to 
increase as the standard systemic therapy for patients with advanced HCC, including HCC with macroscopic vascular invasion $[3,4]$. Sorafenib was shown to significantly improve the overall survival (OS) and disease control rates when compared with placebo, but the tumor response was limited [5, 6]. Transarterial chemoembolization (TACE) is only safe for selected patients because it is associated with an increased risk of ischemic necrosis of the liver and of treatment-related death in patients with PVTT, and its efficacy has remained unsatisfactory [1, 7]. Although hepatic artery infusion chemotherapy (HAIC) has been attempted, it has not showed survival benefit [8].

Although the role of radiotherapy (RT) for HCC had been limited due to the risks of radiation-induced liver disease, recent RT techniques, including three-dimensional conformal radiotherapy (3D-RT), image-guided radiotherapy, and respiratory-gated radiotherapy, as well as information on partial volume liver tolerance, have allowed the delivery of higher radiation doses to the tumor than previously thought possible [1]. Several studies have evaluated the clinical outcomes of RT for inoperable HCC and results showed that RT can produce survival benefits compared to treatment of sorafenib or TACE alone [3, 9-13]. However, because the tumor appearance, irradiation dose, additional treatment such as TACE, and so on are different among the studies, eligible patients and tumors and the appropriate treatment strategy including RT have been unclear.

In this study, we retrospectively evaluated the clinical outcomes in HCC patients treated with RT only for tumor thrombus of hepatic vasculature and bile ducts, and extracted the tumor and treatment factors correlated with the tumor thrombosis response, survival, and cumulative incidence ratio to consider the appropriate treatment for them.

\section{Materials and Methods}

\subsection{Patients}

This multicenter retrospective study was conducted at XXX. The clinical and radiological data of patients who received RT for PVTT, HVTT, IVCTT, or bile duct tumor thrombosis (BDTT) of HCC between 2005 and 2020 were reviewed. The requirement for informed consent was waived because we reviewed anonymous data. The multicenter study approval was obtained from the institutional review board of XXX.

$\mathrm{HCC}$ and tumor thrombosis were diagnosed with dynamic imaging studies, using computed tomography (CT) or magnetic resonance imaging (MRI) with contrast enhancement [4]. Minor portal invasion or portal invasion at the first branch was classified as Vp2-3, and one at the main portal branch was classified as $\mathrm{Vp} 4$. The main hepatic vein invasion was classified as Vv2 and IVCTT as Vv3. BDTT at the first branch was classified as B3 and common hepatic duct tumor thrombosis as B4.

Patients who were diagnosed with a Child-Pugh class C liver function or an Eastern Cooperative Oncology Group (ECOG) performance status (PS) of 4 also had been irradiated with local RT in agreement with the hospital's policy that it would improve the survival even though there is no high-level evidence [4].

\subsection{Treatment}

Image-guided RT was performed in all patients. They received a single daily fraction of 2 or 3 Gy using 6-10 MV $\mathrm{X}$-ray five days per week. Although the $3 \mathrm{~Gy} /$ fraction was often irradiated in XXX, when stereotactic radiotherapy (SRT) was performed, 9-12 Gy/fraction was irradiated. As different doses per fraction were used, the equivalent dose in 2 Gy fractions (EQD2), as the $\alpha / \beta$ ratio of 10 using a linear-quadratic model, was calculated in this study. The gross tumor volume (GTV) included only tumor thrombus in principal, and the primary tumor was partly included in the GTV if the tumor thrombus was close to it. It's not necessary for the primary tumor to be fully irradiated. The clinical target volume (CTV) equaled the GTV. The internal target volume (ITV) was delineated from contrast-enhanced four-dimensional (4D)-CTs, then the planning target volume (PTV) was extended by $5-10 \mathrm{~mm}$ from the ITV. The system of respiratory-gated irradiation was used, and patients received RT while holding their breath. If the tumor was assessed as having little movement due to breathing, it was irradiated at free breathing. Cone-beam CT was performed to ensure the relative position of the diaphragm. If the position was found to be not stable, cone-beam CT was performed daily.

Combined chemotherapy was administered for the tumor thrombosis within three months before the start of RT or after completing RT in this study. Combined local chemotherapy included either TACE or HAIC, and HAIC contained high-dose cisplatin $\left(60 \mathrm{mg} / \mathrm{m}^{2}\right)$ or low-dose cisplatin $(20$ $\mathrm{mg} / \mathrm{m}^{2}$, day 1 and day 8) plus continuous infusion of 5-fluorouracil (350 mg/m², days $1-5$, days 8-12) using a subcutaneous infusion port. Combined systemic treatment included sorafenib, regorafenib, lenvatinib or others.

\subsection{Tumor Thrombosis Evaluation}

Between one to six months after the completion of RT, the size of the tumor thrombus was evaluated using contrast-enhanced CT and/or MRI. On the other hand, the size of the primary tumor was not evaluated because not all the patients were irradiated to the primary tumor. The length of tumor thrombus was precisely measured using a three-dimensional image analysis system (Fujifilm, SYNAPSE VINCENT). We plotted points of the center of tumor thrombus in each axial slices and summed up the lengths between the points (Figure 1). Examples of actual patient images are shown in Figure 2. To eliminate measurement bias, in addition to being measured by radiologists at each of the divisional hospitals, the corresponding author measured again the length of the tumor thrombus in all patients. The definitions of complete response (CR), partial response (PR), stable disease (SD), and progressive disease (PD) were based on the modified Response Evaluation Criteria in Solid Tumors according to 
the previous studies $[1,2]$; CR was defined as a complete disappearance of tumor thrombus; PR as at least a $50 \%$ decrease in the length of thrombus; SD as a $<50 \%$ decrease or $<25 \%$ increase in the length of thrombus; PD as $>25 \%$ increase in the length of thrombus. Patients with a tumor response of $\mathrm{CR}$ or $\mathrm{PR}$ were classified as responders, and patients with tumor response of SD or PD were classified as non-responders.

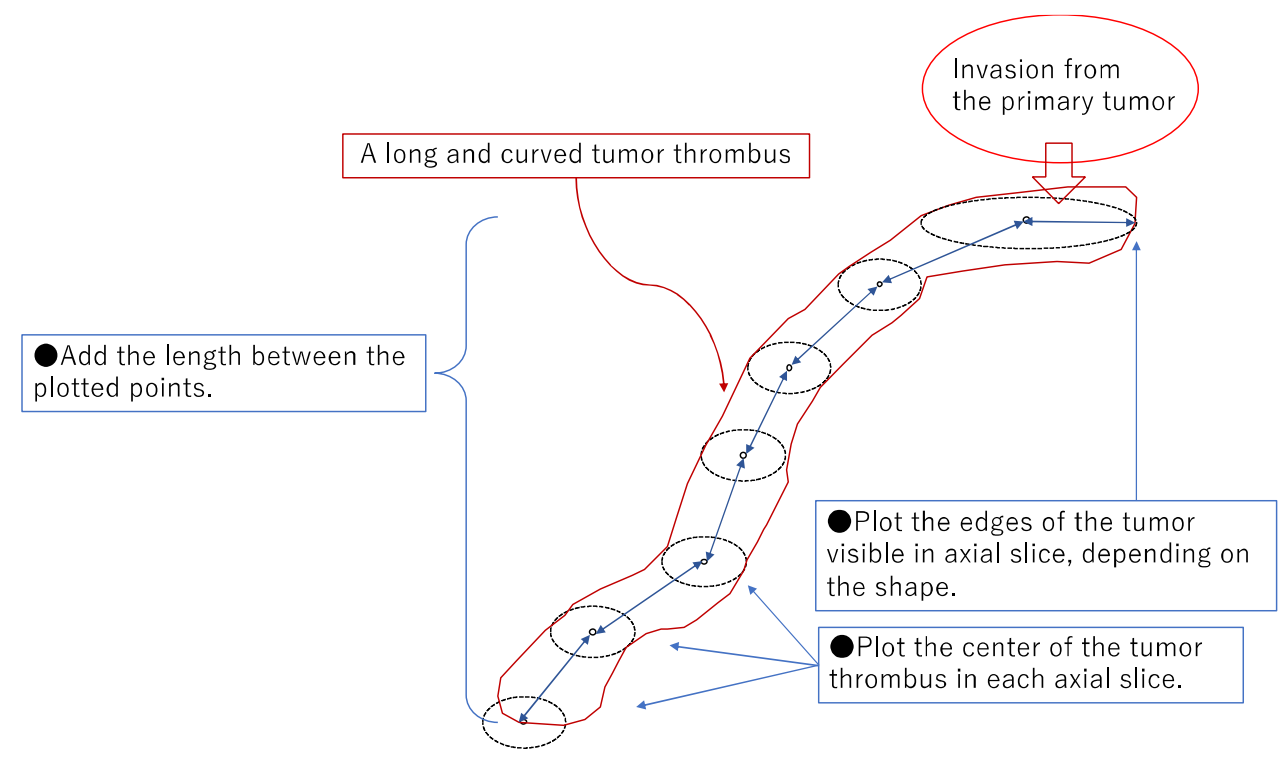

Figure 1. The measurement of the tumor thrombi.
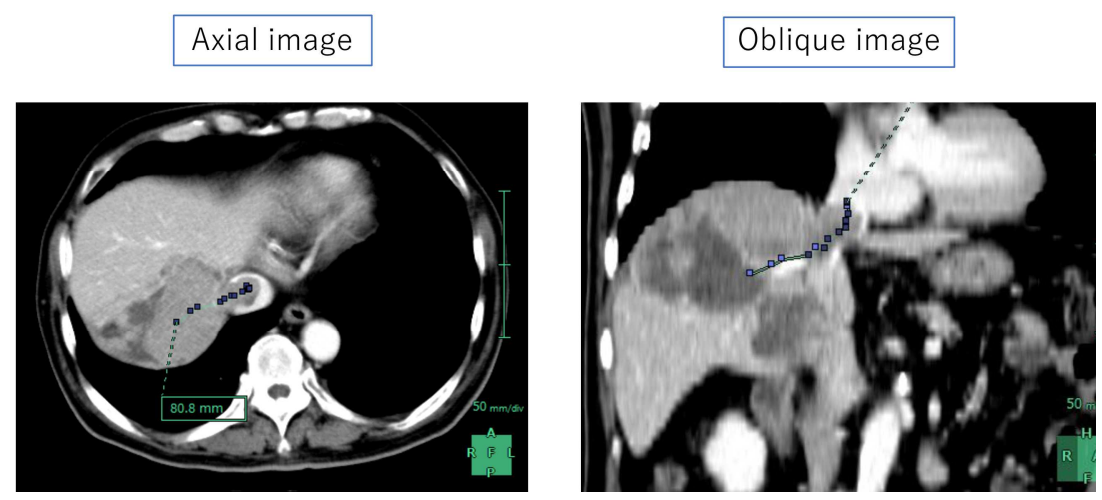

Figure 2. Example of measurement of the tumor thrombus length using a three-dimensional image analysis system (Fujifilm, SYNAPSE VINCENT).

The left figure is an axial slice with the center of the tumor thrombus plotted. And the right figure is an oblique slice that shows most of the plotted points. Not all points are on a single plane because long tumor thrombi are usually curved. Without this system, it would be impossible to accurately measure the length of the tumor thrombus.

\subsection{Liver Toxicity}

Acute adverse effects of the liver occurring within 3 months after RT were graded according to the Common Terminology Criteria for Adverse Events (CTCAE) version 4.0. And acute adverse effects of the liver more than grade 3 was considered as a acute adverse event of the liver after RT. The deterioration in the CPC was also evaluated as late adverse effects after RT. And because the deterioration in the CPC was also caused by the progression of the intrahepatic tumor, cumulative incidence formula (CIF) of the deterioration in the CPC was analyzed in order to evaluate competing risks. We evaluated the irradiated relative volume of the remaining normal liver that received more than 30 Gy (V30Gy). A total irradiated dose of the liver was also calculated as a biologically effective dose 3 (BED3) and an equivalent dose in 2 Gy fractions (EQD2) using a linear-quadratic model with $\alpha / \beta$ ratios of 3 , to evaluate acute and late adverse effects for the liver.

\subsection{Statistics}

The overall survival (OS) was estimated from the date of beginning RT to the date of death of the patient or last contact with the patient. The OS rate was calculated according to the Kaplan-Meier method, and the univariate analysis and multivariate analysis were performed using the log-rank test and a Cox regression model, respectively, to identify prognostic factors. To assess competing risk bias, all patients' causes of death were examined. The objective response rates (ORRs) were tested by using the Fisher analysis in the univariate analysis, and the multivariate analysis was performed using a logistic regression model. The cumulative incidence formulas for the deterioration in the $\mathrm{CPC}$, and the progression of the 
progression of the intrahepatic/extrahepatic tumor were analyzed by using the Fine and Gray regression model.

Receiver operating characteristic (ROC) curve analysis was used to calculate a threshold value for the length of tumor thrombus and size of the primary tumor in relation to objective response. This value was used in analysis of OS, ORRs, and CIF. Values with $p$ values of $<0.05$ by univariate analysis were chosen for multivariate analysis, and $\mathrm{p}$ values less than 0.05 were considered statistically significant.

All the statistical analyses were performed using EZR software, version 1.37 (Saitama Medical Center, Jichi Medical University, Saitama, Japan), which is a graphical user interface for $\mathrm{R}$ (version 2.13.0; The $\mathrm{R}$ Foundation for Statistical Computing, Vienna, Austria). All the statistical analyses were two-sided, and a $p$ value $<0.05$ was considered statistically significant.

\section{Results}

\subsection{Characteristics}

Although 97 patients received RT for PVTT, HVTT, or BDTT during this study's period, 33 patients were excluded as the response evaluation using CT and/or MRI after RT was not carried out in 32 patients, and in one patient there were no data for the clinical characteristics. The remaining 64 patients were enrolled in this study and their clinical characteristics are summarized in Table 1.

The median age of the 64 patients was 71 years (range 51-85 years), and although the viral hepatitis $C$ caused hepatitis in half of them $(n=34)$, non-virus infection caused hepatitis in $37 \%$ of the patients $(n=23)$. PVTT was diagnosed in 34 patients and the main PVTT (Vp4) accounted for $47 \%$ of them $(n=16)$. HVTT was diagnosed in 16 patients and IVCTT (Vv3) accounted for $88 \%$ of them $(n=14)$. Two sites among PVTT, HVTT/IVCTT or BDTT were seen in four patients: both Vv3 and Vp4 in two, both Vv3 and Vp2 in one and both B3 and Vp4 in one. BDTT was diagnosed in nine patients: B3 in four and B4 in five. The median length of tumor thrombus was $3.8 \mathrm{~cm}$ (range $0.8-14.2$ $\mathrm{cm}$ ) and that of the primary tumor was $3.9 \mathrm{~cm}$ (range 0-13.1 $\mathrm{cm})$. A threshold value for the length of tumor thrombus and primary tumor size in relation to tumor thrombus response were $3.2 \mathrm{~cm}$ and $4 \mathrm{~cm}$, respectively. This value was used in the analysis of OS, ORRs and CIF. Lymph-node metastasis was seen in $16 \%(\mathrm{n}=10)$ and distant metastasis was seen in $19 \%$ $(\mathrm{n}=12)$ : lung metastasis in seven, dissemination in two, adrenal metastasis in two, pancreatic metastasis in one and bone metastasis in one.

The median EQD2 of the enrolled patients was 48.75 Gy (range: $32.5-88$ Gy). Actually, 31 patients received 45 Gy in 15 fractions, 21 received 39 Gy in 13 fractions, 4 received 50 Gy in 25 fractions, 2 received 60 Gy in 30 fractions, 1 received 30 Gy in 10 fractions, 1 received 56 Gy in 28 fractions, and 3 received SRT of 48-54 Gy in 4-6 fractions (Table 2).

Combined chemotherapy was administered to 39 patients. Local chemotherapy was undergone by 25 patients: TACE in 13 and HAIC in 13. Systemic treatment with sorafenib was administered to 14 patients, and lenvatinib was to 3 . Other systemic anticancer drugs were used in 4 patients, and one of them also received TACE.

Table 1. Summary of clinical characteristics.

\begin{tabular}{|c|c|c|}
\hline Characteristic $(n=64)$ & Median (range)/ no. of patients & $(\%)$ \\
\hline \multicolumn{3}{|l|}{ Gender } \\
\hline Male & 50 & 78 \\
\hline Female & 14 & 22 \\
\hline Age (years) & $71(51-85)$ & \\
\hline \multicolumn{3}{|l|}{ ECOG PS } \\
\hline $0-1$ & 54 & 84 \\
\hline $2-3$ & 9 & 14 \\
\hline 4 & 1 & 2 \\
\hline \multicolumn{3}{|l|}{ Etiology of hepatitis } \\
\hline Hepatitis B virus & 7 & 11 \\
\hline Hepatitis $\mathrm{C}$ virus & 29 & 45 \\
\hline $\mathrm{B}$ or $\mathrm{C}$ virus and Alcohol & 5 & 8 \\
\hline Alcohol & 10 & 16 \\
\hline NASH & 5 & 8 \\
\hline Others & 8 & 13 \\
\hline \multicolumn{3}{|l|}{ Child-Pugh class } \\
\hline A & 40 & 63 \\
\hline B & 22 & 34 \\
\hline $\mathrm{C}$ & 2 & 3 \\
\hline \multicolumn{3}{|l|}{ Tumor thrombosis invasion site } \\
\hline Vp2 & 2 & 5 \\
\hline Vp3 & 16 & 25 \\
\hline Vp4 & 16 & 25 \\
\hline Vv2 & 2 & 3 \\
\hline Vv3 & 14 & 22 \\
\hline 2 sites (Vp, Vv or Bile duct) & 4 & 6 \\
\hline Bile duct & 9 & 14 \\
\hline \multicolumn{3}{|l|}{$\mathrm{T}^{*}$} \\
\hline T2 & 11 & 17 \\
\hline T3 & 2 & 3 \\
\hline T4 & 51 & 80 \\
\hline \multicolumn{3}{|l|}{$\mathrm{N}^{*}$} \\
\hline No & 54 & 84 \\
\hline N1 & 10 & 16 \\
\hline \multicolumn{3}{|l|}{$\mathrm{M}^{*}$} \\
\hline M0 & 52 & 81 \\
\hline M1 & 12 & 19 \\
\hline \multicolumn{3}{|l|}{ Staging* } \\
\hline II & 8 & 13 \\
\hline IIIa & 1 & 2 \\
\hline IIIlb & 36 & 56 \\
\hline $\mathrm{IVa}$ & 7 & 11 \\
\hline $\mathrm{IVb}$ & 12 & 19 \\
\hline \multicolumn{3}{|l|}{ Length of tumor thrombus $(\mathrm{cm})$} \\
\hline$<3.2$ & 43 & 67 \\
\hline$\geq 3.2$ & 21 & 33 \\
\hline \multicolumn{3}{|l|}{ Primary tumor size $(\mathrm{cm})$} \\
\hline$<4$ & 35 & 55 \\
\hline$\geq 4$ & 29 & 45 \\
\hline
\end{tabular}

NASH, Non-alcoholic steatohepatitis; Vv2, Presence of a tumor thrombus in a major hepatic vein; Vv3, Presence of a tumor thrombus in inferior vena cava; $\mathrm{Vp} 2$, Presence of a tumor thrombus in the second-order branches of the portal vein; Vp3, Presence of a tumor thrombus in the first- order branches of the portal vein; Vp4, Presence of a tumor thrombus in the main trunk of the portal vein or a portal vein, branch contralateral to the primarily involved lobe (or both)

*Staging of hepatocellular carcinoma was performed according to the 8th edition of the Union for International Cancer Control (UICC)-Tumor Node Metastasis (TNM) classification. 
Table 2. Treatment characteristics.

\begin{tabular}{lll}
\hline Treatment Characteristic (n=64) & $\begin{array}{l}\text { Median (range)/ } \\
\text { no. of patients }\end{array}$ & (\%) \\
\hline Chemotherapy (Beofre RT/After RT) & & \\
TACE/None or None/TACE & 9 & 14 \\
HAIC/None or None/HAIC & 8 & 13 \\
TACE/HAIC & 1 & 2 \\
Sorafenib/Sorafenib or None/Sorafenib & 9 & 14 \\
HAIC/Sorafenib & 3 & 5 \\
TACE/Sorafenib or Sorafenib/TACE & 2 & 3 \\
TACE/Other agents & 1 & 2 \\
Lenvatinib/Lenvatinib or None/Lenvatinib & 2 & 3 \\
Lenvatinib/HAIC & 1 & 2 \\
Other agents/Other agents or None/Other agents & 3 & 5 \\
None & 25 & 39 \\
EQD2 (Gy) & & \\
32.5 & 1 & 2 \\
41.67 & 1 & 2 \\
42.35 & 21 & 33 \\
48.75 & 31 & 48 \\
50 & 4 & 6 \\
56 & 1 & 2 \\
60 & 2 & 3 \\
$\geq 83$ (SRT) & 3 & 5 \\
Brradiated sites & & 25 \\
Only tumor thrombus & 16 & 75 \\
\hline
\end{tabular}

EQD2, Equivalent dose in 2-Gy fractions; RT, radiation therapy; HAIC, Hepatic arterial infusion chemotherapy; TACE, Transarterial chemoembolization.

\subsection{Overall Survival}

The median follow-up period for all the patients was 8 months (range 2-90 months), and the median survival time was 9 months. The one- and three-year OS rates were $44 \%$ and $17 \%$, respectively (Figure 3). Thirty-nine patients died from the disease. Six patients died of acute causes: esophageal variceal rupture in 4 , asphyxiation in 1 , and a perioperative infection in 1. Ten patients were alive until the statistics were discontinued. Ten patients lost their follow-up.

In the univariate analysis the ECOG PS $(p=0.01)$, Child-Pugh class $(p=0.003)$, primary tumor size $(p=0.009)$, length of tumor thrombus $(\mathrm{p}=0.01)$, EQD2 $(\mathrm{p}=0.001)$, combined chemotherapy $(\mathrm{p}=0.04)$, response of the tumor thrombus $(p=0.005)$, and irradiated sites $(p=0.04)$ were significantly related with OS (Table 3 ). Multivariate analyses were performed among these six important factors, and Child-Pugh class (HR 12, 95\% CI $[2.8,5.8], \mathrm{p}=0.000002$ ), primary tumor size (HR 5.2, 95\% CI [1.4, 2.7], $\mathrm{p}=0.003$ ), and response of the tumor thrombus (HR $0.5,95 \% \mathrm{CI}[0.1,0.3]$, $\mathrm{p}=0.0002$ ) were shown as unique significant factors. Age, $\mathrm{N}$ stage, $\mathrm{M}$ stage, length of the primary tumor, or grade and site of tumor thrombosis were not related to the OS.

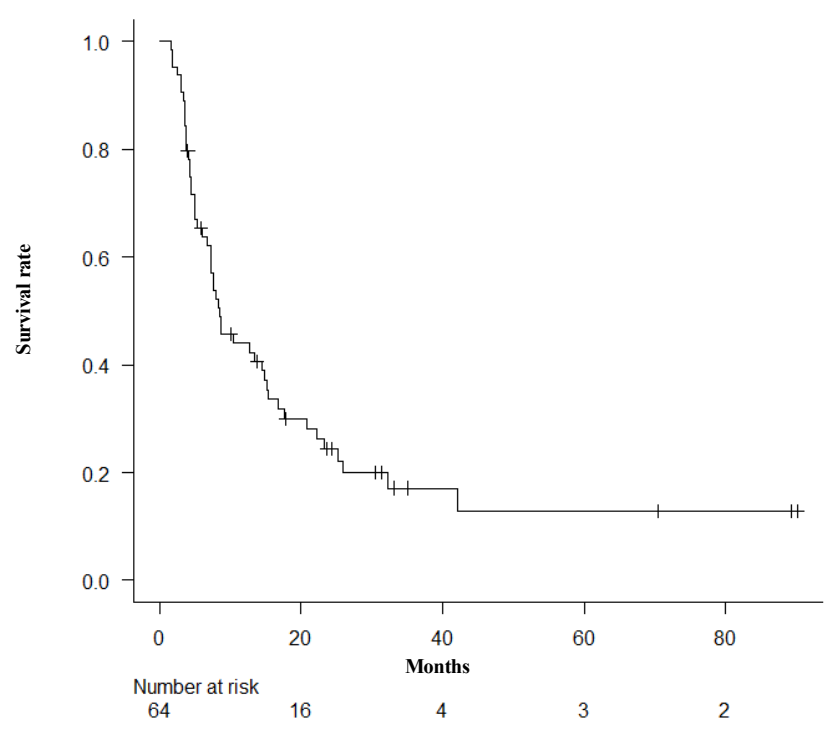

Figure 3. Overall survival of all the patients.

The median survival time was 9 months for all the patients. The one- and three-year OS rates were $44 \%$ and $17 \%$, respectively.

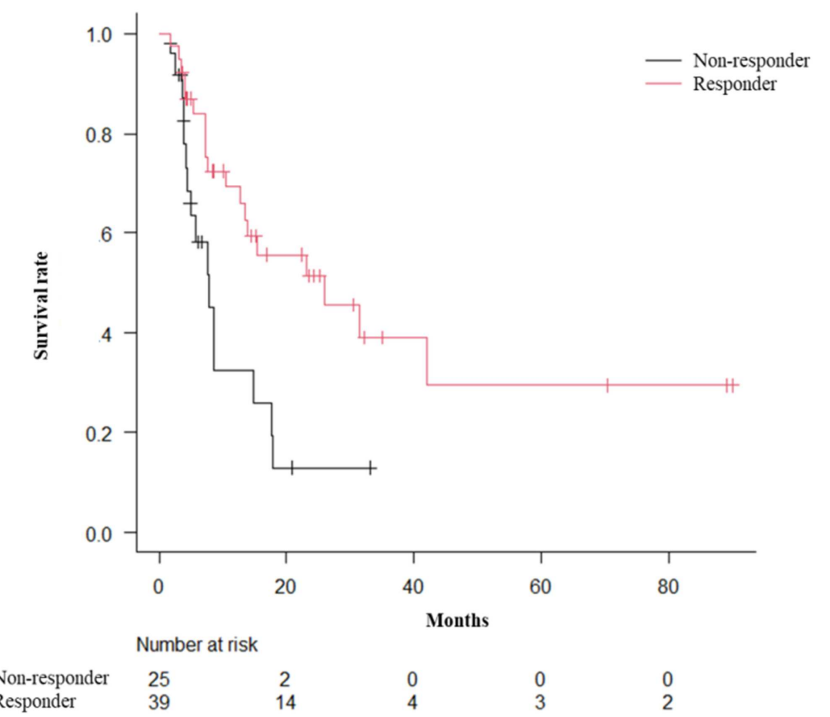

Figure 4. Overall survival between the responders and the non-responders groups.

The one-year survival rate of the responder and non-responder groups was $69 \%$ and $32 \%$, respectively $(\mathrm{p}=0.005)$. Responders had significantly better OS than non-responders.

Table 3. Univariate and multivariate analysis of the prognostic factors influencing the OS.

\begin{tabular}{lllll}
\hline \multirow{2}{*}{ Prognostic factor } & \multirow{2}{*}{ No. } & \multicolumn{2}{c}{ Univariate analysis } & Multivariate analysis \\
\cline { 3 - 5 } & & 1-year OS (\%) & P-value & HR (95\% CI) \\
\hline Gender & 50 & 60 & 0.7 & \\
Male & 14 & 5 & & \\
Female & & & \\
Age (y) & & & \\
\hline
\end{tabular}


Takuya Nagano et al.: Clinical Results of Radiotherapy That Targeted for Tumor Thrombi from

Hepatocellular Carcinoma: A Multicenter Retrospective Study

\begin{tabular}{|c|c|c|c|c|c|}
\hline \multirow{2}{*}{ Prognostic factor } & \multirow{2}{*}{ No. } & \multicolumn{2}{|c|}{ Univariate analysis } & \multicolumn{2}{|c|}{ Multivariate analysis } \\
\hline & & 1-year OS (\%) & P-value & HR $(95 \%$ CI $)$ & P-value \\
\hline$<60$ & 9 & 51 & 0.7 & & \\
\hline$\geq 60$ & 55 & 57 & & & \\
\hline \multicolumn{6}{|l|}{ ECOG PS } \\
\hline $0-1$ & 54 & 64 & 0.01 & $1.6(0.3-0.7)$ & 0.4 \\
\hline $2-4$ & 10 & 24 & & & \\
\hline \multicolumn{6}{|l|}{ Child-Pugh class } \\
\hline A & 39 & 65 & 0.003 & $12(2.8-5.8)$ & 0.000002 \\
\hline $\mathrm{B}-\mathrm{C}$ & 25 & 42 & & & \\
\hline \multicolumn{6}{|l|}{ Grade of tumor thorombosis } \\
\hline Vv2 or Vp2-3 or BDTT & 19 & 60 & 0.9 & & \\
\hline Vv3 or Vp4 & 45 & 55 & & & \\
\hline \multicolumn{6}{|l|}{ Site of tumor thorombosis } \\
\hline PVTT & 34 & 47 & 0.4 & & \\
\hline HVTT/IVCTT & 17 & 73 & & & \\
\hline Both PVTT and HVTT/IVCTT & 4 & 33 & & & \\
\hline BDTT & 9 & 75 & & & \\
\hline \multicolumn{6}{|l|}{ Presense of BDTT } \\
\hline Yes & 9 & 56 & 0.8 & & \\
\hline No & 55 & 42 & & & \\
\hline \multicolumn{6}{|l|}{$\mathrm{T}$} \\
\hline $\mathrm{T} 2$ & 11 & 100 & 0.05 & & \\
\hline $\mathrm{T} 3$ & 2 & NA & & & \\
\hline $\mathrm{T} 4$ & 51 & 49 & & & \\
\hline \multicolumn{6}{|l|}{$\mathrm{N}$} \\
\hline No & 54 & 54 & 0.9 & & \\
\hline N1 & 10 & 69 & & & \\
\hline \multicolumn{6}{|l|}{ M } \\
\hline M0 & 52 & 54 & 0.4 & & \\
\hline M1 & 12 & 72 & & & \\
\hline \multicolumn{6}{|l|}{ TNM staging } \\
\hline II & 8 & 100 & 0.3 & & \\
\hline IIIa & 1 & NA & & & \\
\hline IIIb & 36 & 44 & & & \\
\hline IVa & 7 & 57 & & & \\
\hline $\mathrm{IVb}$ & 12 & 72 & & & \\
\hline \multicolumn{6}{|l|}{ Primary tumor size $(\mathrm{cm})$} \\
\hline$<4$ & 35 & 73 & 0.009 & $5.2(1.4-2.7)$ & 0.003 \\
\hline$\geq 4$ & 29 & 35 & & & \\
\hline \multicolumn{6}{|l|}{ Length of tumor thrombosis (cm) } \\
\hline$<3.2$ & 43 & 64 & 0.01 & $1.5(0.4-0.7)$ & 0.4 \\
\hline$\geq 3.2$ & 21 & 42 & & & \\
\hline \multicolumn{6}{|l|}{ EQD2 (Gy) } \\
\hline$<48.75$ & 23 & 37 & 0.001 & $1(1.4-2.7)$ & 0.06 \\
\hline$\geq 48.75$ & 41 & 67 & & & \\
\hline \multicolumn{6}{|c|}{ Combined CTX with RT (Local or Systemic) } \\
\hline Yes & 39 & 63 & 0.04 & & \\
\hline No & 25 & 47 & & & \\
\hline Acute adverse event & & & & & \\
\hline Yes & 9 & 54 & 0.5 & & \\
\hline No & 55 & 89 & & & \\
\hline Response & & & & & \\
\hline Yes & 39 & 69 & 0.005 & $0.5(0.1-0.25)$ & 0.0002 \\
\hline No & 25 & 32 & & & \\
\hline Irradiated sites & & & & & \\
\hline Both primary tumor and its thrombus & 18 & 63 & 0.04 & & \\
\hline Only tumor thrombus & 48 & 38 & & & \\
\hline
\end{tabular}

OS, Overall survival; HR, Hazard ratio; ECOG-PS, Eastern Cooperative Oncology Group Performance Status; BDTT, Bile duct tumor thrombosis; PVTT, Portal vein tumor thrombosis; IVCTT, Inferior vena cava tumor thrombosis; HVTT, Hepatic vein tumor thrombosis; Vv2, Presence of a tumor thrombus in a major hepatic vein; Vv3, Presence of a tumor thrombus in inferior vena cava; Vp2, Presence of a tumor thrombus in the second-order branches of the portal vein; Vp3, Presence of a tumor thrombus in the first- order branches of the portal vein; Vp4, Presence of a tumor thrombus in the main trunk of the portal vein or a portal vein, branch contralateral to the primarily involved lobe (or both); EQD2, Equivalent dose in 2-Gy fractions; CTX, Chemotherapy; RT, radiation therapy; HAIC, Hepatic arterial infusion chemotherapy; TACE, Transarterial chemoembolization. 


\subsection{Tumor Response}

Of the 64 patients, 27 patients (42\%) achieved CR, 12 patients (19\%) achieved PR, 22 patients (34\%) had SD, and 3 patients had PD (5\%). The ORR of all the patients was $61 \%$.

The ORRs of patients who received an EQD2 of $<48.75 \mathrm{~Gy}$ or $>=48.75$ Gy were $44 \%$ and $71 \%(p=0.04)$. In the univariate analysis, EQD2 was only significantly related to the tumor response (Table 4). In the multivariate analysis, no factors were found to be significantly different. The one-year OS rates of responders $(n=39)$ and non-responders $(n=25)$ were $69 \%$ and $32 \%$, respectively, and the difference was statistically significant $(\mathrm{p}=0.005$, Figure 3$)$.

Table 4. Response of tumor thrombosis.

\begin{tabular}{|c|c|c|c|c|}
\hline & \multicolumn{2}{|l|}{ Univariate analysis } & \multicolumn{2}{|l|}{ Multivariate analysis } \\
\hline & Odds ratio (95\% CI) & P-value & Odds ratio (95\% CI) & P-value \\
\hline \multicolumn{5}{|l|}{ ECOG PS } \\
\hline $0-1$ & $0.59(0.11-2.9)$ & 0.5 & & \\
\hline \multicolumn{5}{|l|}{$2-4$} \\
\hline \multicolumn{5}{|l|}{ Cause of hepatitis } \\
\hline $\begin{array}{l}\text { Viral hepatitis B or C } \\
\text { others }\end{array}$ & $0.54(0.17-1.7)$ & 0.3 & & \\
\hline \multicolumn{5}{|l|}{ Child-Pugh class } \\
\hline A & $1.23(0.39-4)$ & 0.8 & & \\
\hline \multicolumn{5}{|l|}{ B-C } \\
\hline \multicolumn{5}{|l|}{ Site } \\
\hline $\mathrm{Vv} 2$ or $\mathrm{Vp} 2-3$ or $\mathrm{BD}$ & $0.87(0.24-3)$ & 1 & & \\
\hline \multicolumn{5}{|l|}{ Vv3 or Vp4 } \\
\hline \multicolumn{5}{|l|}{$\mathrm{T}$} \\
\hline $\mathrm{T} 2-3$ & $0.23(0.02-1.19)$ & 0.06 & $1.13(0.037-0.21)$ & 0.07 \\
\hline \multicolumn{5}{|l|}{$\mathrm{T} 4$} \\
\hline \multicolumn{5}{|l|}{$\mathrm{N}$} \\
\hline No & $0.59(0.11-2.92)$ & 0.5 & & \\
\hline \multicolumn{5}{|l|}{ N1 } \\
\hline \multicolumn{5}{|l|}{ M } \\
\hline M0 & $0.58(0.13-2.51)$ & 0.5 & & \\
\hline \multicolumn{5}{|l|}{ M1 } \\
\hline \multicolumn{5}{|l|}{ Primary tumor size $(\mathrm{cm})$} \\
\hline$<4<$ & $0.64(0.2-1.98)$ & 0.5 & $4.67(0.4-1.4)$ & 0.6 \\
\hline \multicolumn{5}{|l|}{$\geq 4$} \\
\hline \multicolumn{5}{|l|}{ Length of tumor thrombosis (cm) } \\
\hline$<3.2$ & $0.44(0.13-1.45)$ & 0.2 & $1.8(0.15-0.5)$ & 0.3 \\
\hline \multicolumn{5}{|l|}{$\geq 3.2$} \\
\hline \multicolumn{5}{|l|}{ EQD2 (Gy) } \\
\hline$<48.75$ & $3.1(0.95-10.4)$ & 0.04 & $8.5(0.9-2.9)$ & 0.08 \\
\hline \multicolumn{5}{|c|}{$\geq 48.75$} \\
\hline \multicolumn{5}{|c|}{ Combined CTX with RT (Local or Systemic CTX) } \\
\hline Yes & $1.39(0.44-4.4)$ & 0.6 & & \\
\hline \multicolumn{5}{|l|}{ No } \\
\hline Irradiated sites & & & & \\
\hline $\begin{array}{l}\text { Both primary tumor and its thrombus } \\
\text { Only tumor thrombus }\end{array}$ & $0.64(0.15-2.4)$ & 0.6 & $3.7(0.25-0.96)$ & 1.0 \\
\hline
\end{tabular}

CI, Confidence interval; ECOG-PS, Eastern Cooperative Oncology Group Performance Status; Vv2, Presence of a tumor thrombus in a major hepatic vein; Vv3, Presence of a tumor thrombus in inferior vena cava; Vp2, Presence of a tumor thrombus in the second-order branches of the portal vein; Vp3, Presence of a tumor thrombus in the first- order branches of the portal vein; Vp4, Presence of a tumor thrombus in the main trunk of the portal vein or a portal vein, branch contralateral to the primarily involved lobe (or both); EQD2, Equivalent dose in 2-Gy fractions; CTX, Chemotherapy; RT, radiation therapy; HAIC, Hepatic arterial infusion chemotherapy; TACE, Transarterial chemoembolization.

\subsection{Liver Toxicities and Cumulative Incidence Analysis}

Nine patients (14\%) were observed to have elevated levels of AST/ALT of more than Grade 3. One of them suffered from rupture of esophageal varices because of liver cirrhosis and acute liver failure of Grade 4. One suffered from a pulmonary embolism and an unknown fever. Two suffered acute liver failure after treatment of TACE or HAIC. Two died of disease progression two months after the elevated level of AST/ALT. The other three patients recovered from acute liver toxicity for about 10 days without any reason or events.
Deterioration in the Child-Pugh class was observed in 21 patients $(48 \%)$. On the other hand, 31 patients $(33 \%)$ died or lost to follow-up and 12 patients (19\%) survived without liver damage. Because it was not known whether their liver failures were due to disease progression or adverse events, we evaluated CIF for the deterioration in the Child-Pugh class and the progression of intrahepatic/extrahepatic tumors.

In the univariate analysis, an event of acute liver failure was only significantly related to the deterioration in the Child-Pugh class (HR 3.1, 95\% CI [1.3, 7.6], $\mathrm{p}=0.001$ ). In the multivariate 
Takuya Nagano et al.: Clinical Results of Radiotherapy That Targeted for Tumor Thrombi from Hepatocellular Carcinoma: A Multicenter Retrospective Study

analysis, event of acute liver failure was either only

(HR 3.3, 95\% CI $[1.3,8.2], \mathrm{p}=0.01$, Table 5). significantly related to the deterioration in the Child-Pugh class

Table 5. Cumulative incidence for the deterioration of Child-Pugh class between the factors.

\begin{tabular}{|c|c|c|c|c|}
\hline & \multicolumn{2}{|l|}{ Univariate analysis } & \multicolumn{2}{|l|}{ Multivariate analysis } \\
\hline & Hazard ratio $(95 \% \mathrm{CI})$ & P-value & Hazard ratio $(95 \% \mathrm{CI})$ & P-value \\
\hline \multicolumn{5}{|l|}{ ECOG PS } \\
\hline $0-1$ & $2.1(0.8-5.8)$ & 0.2 & & \\
\hline \multicolumn{5}{|l|}{$2-4$} \\
\hline \multicolumn{5}{|l|}{ Cause of hepatitis } \\
\hline Viral hepatitis B or C & $0.6(0.2-1.5)$ & 0.3 & & \\
\hline \multicolumn{5}{|l|}{ others } \\
\hline Child-Pugh class & & & $0.7(0.3-1.98)$ & 0.5 \\
\hline A & $0.8(0.3-2.1)$ & 0.7 & & \\
\hline \multicolumn{5}{|l|}{ B-C } \\
\hline \multicolumn{5}{|l|}{ Site } \\
\hline $\mathrm{Vv} 2$ or $\mathrm{Vp} 2-3$ or $\mathrm{BD}$ & $1.4(0.5-3.9)$ & 0.5 & & \\
\hline \multicolumn{5}{|l|}{$\mathrm{Vv} 3$ or $\mathrm{Vp} 4$} \\
\hline \multicolumn{5}{|l|}{$\mathrm{T}$} \\
\hline $\mathrm{T} 2-3$ & $1.6(0.4-5.7)$ & 0.5 & & \\
\hline \multicolumn{5}{|l|}{$\mathrm{T} 4$} \\
\hline \multicolumn{5}{|l|}{$\mathrm{N}$} \\
\hline N0 & $0.9(0.3-3.1)$ & 0.9 & & \\
\hline \multicolumn{5}{|l|}{ N1 } \\
\hline \multicolumn{5}{|l|}{ M } \\
\hline M0 & $0.9(0.4-2.5)$ & 1 & & \\
\hline \multicolumn{5}{|l|}{ M1 } \\
\hline \multicolumn{5}{|l|}{ Primary tumor size $(\mathrm{cm})$} \\
\hline$<4$ & $1.8(0.8-4.2)$ & 0.2 & & \\
\hline \multicolumn{5}{|l|}{$>4$} \\
\hline \multicolumn{5}{|l|}{ Length of tumor thrombosis (cm) } \\
\hline$<3.2$ & $1.3(0.6-3.1)$ & 0.5 & & \\
\hline \multicolumn{5}{|l|}{$\geq 3.2$} \\
\hline \multicolumn{5}{|l|}{ EQD2 (Gy) } \\
\hline$<48.75$ & $1.2(0.4-2.9)$ & 0.7 & $1.2(0.3-4)$ & 0.8 \\
\hline \multicolumn{5}{|c|}{$\geq 48.75$} \\
\hline \multicolumn{5}{|c|}{ Combined CTX with RT (Local or Systemic CTX) } \\
\hline Yes & $1.2(0.5-3.1)$ & 0.7 & $0.96(0.28-3.3)$ & 1.0 \\
\hline \multicolumn{5}{|l|}{ No } \\
\hline \multicolumn{5}{|l|}{ Acute adverse events } \\
\hline Yes & $3.1(1.3-7.6)$ & 0.001 & $3.3(1.3-8.2)$ & 0.01 \\
\hline \multicolumn{5}{|l|}{ No } \\
\hline Response & & & & \\
\hline Yes & $1.6(0.6-4.2)$ & 0.3 & $1.5(0.54-4.1)$ & 0.5 \\
\hline No & & & & \\
\hline Irradiated sites & & & & \\
\hline Both primary tumor and its thrombus & $0.6(0.2-1.8)$ & 0.4 & & \\
\hline Only tumor thrombus & & & & \\
\hline
\end{tabular}

CI, Confidence interval; ECOG-PS, Eastern Cooperative Oncology Group Performance Status; Vv2, Presence of a tumor thrombus in a major hepatic vein; Vv3, Presence of a tumor thrombus in inferior vena cava; Vp2, Presence of a tumor thrombus in the second-order branches of the portal vein; Vp3, Presence of a tumor thrombus in the first- order branches of the portal vein; Vp4, Presence of a tumor thrombus in the main trunk of the portal vein or a portal vein, branch contralateral to the primarily involved lobe (or both); EQD2, Equivalent dose in 2-Gy fractions; CTX, Chemotherapy; RT, radiation therapy; HAIC, Hepatic arterial infusion chemotherapy; TACE, Transarterial chemoembolization.

The progression of intrahepatic tumors was observed in 39 patients $(61 \%)$. On the other hand, 22 patients $(34 \%)$ died or lost to follow-up and 3 patients $(5 \%)$ survived without the progression of intrahepatic tumors.

In the univariate analysis, the length of TT was only significantly related to the progression of intrahepatic tumors (HR 0.4, 95\% CI [0.2, 0.9], $\mathrm{p}=0.03$ ). In the multivariate analysis, the length of TT was either only significantly related to the progression of intrahepatic tumors (HR $0.3,95 \%$ CI [0.1, 0.9 ], $\mathrm{p}=0.03$, Table 6). 
Table 6. Cumulative incidence for progression of the intrahepatic tumor between the factors.

\begin{tabular}{|c|c|c|c|c|}
\hline & \multicolumn{2}{|l|}{ Univariate analysis } & \multicolumn{2}{|l|}{ Multivariate analysis } \\
\hline & Hazard ratio $(95 \% \mathrm{CI})$ & P-value & Hazard ratio $(95 \% \mathrm{CI})$ & P-value \\
\hline \multicolumn{5}{|l|}{ ECOG PS } \\
\hline $0-1$ & $1(0.4-2.6)$ & 0.9 & & \\
\hline \multicolumn{5}{|l|}{$2-4$} \\
\hline \multicolumn{5}{|l|}{ Cause of hepatitis } \\
\hline $\begin{array}{l}\text { Viral hepatitis B or C } \\
\text { others }\end{array}$ & $1(0.5-1.8)$ & 1 & & \\
\hline \\
\hline A & $0.5(0.2-1.1)$ & 0.09 & $0.6(0.2-1.5)$ & 0.3 \\
\hline \multicolumn{5}{|l|}{ B-C } \\
\hline \multicolumn{5}{|l|}{ Site } \\
\hline $\mathrm{Vv} 2$ or $\mathrm{Vp} 2-3$ or $\mathrm{BD}$ & $0.8(0.4-1.5)$ & 0.4 & & \\
\hline \multicolumn{5}{|l|}{ Vv3 or Vp4 } \\
\hline \multicolumn{5}{|l|}{$\mathrm{T}$} \\
\hline $\mathrm{T} 2-3$ & $1.4(0.6-3)$ & 0.4 & & \\
\hline \multicolumn{5}{|l|}{$\mathrm{T} 4$} \\
\hline \multicolumn{5}{|l|}{$\mathrm{N}$} \\
\hline N0 & $0.5(0.2-1.5)$ & 0.2 & & \\
\hline \multicolumn{5}{|l|}{ N1 } \\
\hline \multicolumn{5}{|l|}{ M } \\
\hline M0 & $0.9(0.4-1.8)$ & 0.7 & & \\
\hline \multicolumn{5}{|l|}{ M1 } \\
\hline \multicolumn{5}{|l|}{ Primary tumor size $(\mathrm{cm})$} \\
\hline$<4$ & $0.7(0.4-1.4)$ & 0.4 & $1.1(0.5-2.5)$ & 0.8 \\
\hline \multicolumn{5}{|l|}{$\geq 4$} \\
\hline \multicolumn{5}{|l|}{ Length of tumor thrombosis (cm) } \\
\hline$<3.2$ & $0.4(0.2-0.9)$ & 0.03 & $0.3(0.1-0.9)$ & 0.03 \\
\hline \multicolumn{5}{|l|}{$\geq 3.2$} \\
\hline \multicolumn{5}{|l|}{ EQD2 (Gy) } \\
\hline$<48.75 \mathrm{~Gy}$ & $0.8(0.4-1.6)$ & 0.6 & & \\
\hline \multicolumn{5}{|l|}{$\geq 48.75$} \\
\hline \multicolumn{5}{|c|}{ Combined CTX with RT (Local or Systemic CTX) } \\
\hline Yes & $1.1(0.6-2.1)$ & 0.8 & & \\
\hline \multicolumn{5}{|l|}{ No } \\
\hline \multicolumn{5}{|l|}{ Acute adverse events } \\
\hline Yes & $0.6(0.2-1.5)$ & 0.3 & & \\
\hline \multicolumn{5}{|l|}{ No } \\
\hline \multicolumn{5}{|l|}{ Response } \\
\hline Yes & $0.7(0.4-1.3)$ & 0.3 & $0.5(0.3-1.1)$ & 0.08 \\
\hline No & & & & \\
\hline Irradiated sites & & & & \\
\hline $\begin{array}{l}\text { Both primary tumor and its thrombus } \\
\text { Only tumor thrombus }\end{array}$ & $1.8(0.96-3.3)$ & 0.07 & $1.6(0.7-3.5)$ & 0.3 \\
\hline
\end{tabular}

The progression of extrahepatic tumors was observed in 25 patients $(39 \%)$. On the other hand, 8 patients $(13 \%)$ died or lost to follow-up and 31 patients $(48 \%)$ survived without the progression of extrahepatic tumors.

In the univariate analysis, M1 was only significantly related to the progression of extrahepatic tumors (HR 6.5, 95\% CI $[2.8,15], \mathrm{p}=0.0002)$. In the multivariate analysis, M1 was either only significantly related to the progression of extrahepatic tumors (HR 5.6, 95\% CI [2.6, 11.9], p < 0.00001, Table 7).

Table 7. Cumulative incidence for progression of the extrahepatic c tumor between the factors.

\begin{tabular}{|c|c|c|c|c|}
\hline & \multicolumn{2}{|l|}{ Univariate analysis } & \multicolumn{2}{|l|}{ Multivariate analysis } \\
\hline & Hazard ratio (95\% CI) & P-value & Hazard ratio (95\% CI) & P-value \\
\hline \multicolumn{5}{|l|}{ ECOG PS } \\
\hline $0-1$ & $0.4(0.1-1.7)$ & 0.2 & & \\
\hline \multicolumn{5}{|l|}{$2-4$} \\
\hline \multicolumn{5}{|l|}{ Cause of hepatitis } \\
\hline \multicolumn{5}{|l|}{ others } \\
\hline A & $0.4(0.1-0.98)$ & 0.04 & $0.4(0.1-1.1)$ & 0.6 \\
\hline \multicolumn{5}{|l|}{ B-C } \\
\hline \multicolumn{5}{|l|}{ Site } \\
\hline $\mathrm{Vv} 2$ or $\mathrm{Vp} 2-3$ or $\mathrm{BD}$ & $2.1(0.8-5.2)$ & 0.1 & & \\
\hline
\end{tabular}




\begin{tabular}{|c|c|c|c|c|}
\hline & \multicolumn{2}{|l|}{ Univariate analysis } & \multicolumn{2}{|l|}{ Multivariate analysis } \\
\hline & Hazard ratio $(95 \% \mathrm{CI})$ & P-value & Hazard ratio $(95 \% \mathrm{CI})$ & P-value \\
\hline \multicolumn{5}{|l|}{$\mathrm{Vv} 3$ or $\mathrm{Vp} 4$} \\
\hline $\mathrm{T} 2-3$ & $7.9(1.1-58)$ & 0.04 & $4.8(0.6-37.9)$ & 0.1 \\
\hline \multicolumn{5}{|l|}{$\mathrm{T} 4$} \\
\hline $\mathrm{N}$ & & & & \\
\hline \multirow{2}{*}{\multicolumn{5}{|c|}{$1.1(0.1-1.0)$}} \\
\hline & & & & \\
\hline \multicolumn{5}{|l|}{ M } \\
\hline $\begin{array}{l}\text { M0 } \\
\text { M1 }\end{array}$ & $6.5(2.8-15)$ & $<0.001$ & $5.6(2.6-11.9)$ & $<0.001$ \\
\hline \multicolumn{5}{|l|}{ Primary tumor size $(\mathrm{cm})$} \\
\hline$<4$ & $1.2(0.6-2.7)$ & 0.6 & & \\
\hline \multicolumn{4}{|l|}{ 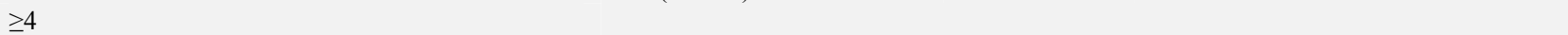 } & \\
\hline $\begin{array}{l}<3.2 \\
\geq 3.2\end{array}$ & $1.1(0.5-2.6)$ & 0.8 & & \\
\hline \multicolumn{5}{|l|}{ EQD2 (Gy) } \\
\hline$\geq 48.75$ & $1.1(0.5-2.6)$ & 0.8 & & \\
\hline \multicolumn{5}{|c|}{ Combined CTX with RT (Local or Systemic CTX) } \\
\hline \multicolumn{4}{|l|}{ No } & \\
\hline \multicolumn{5}{|l|}{ Acute adverse events } \\
\hline $\begin{array}{l}\text { Yes } \\
\text { No }\end{array}$ & $1.7(0.7-4.2)$ & 0.3 & & \\
\hline \multicolumn{5}{|l|}{ Response } \\
\hline \multicolumn{4}{|l|}{ No } & \\
\hline Irradiated sites & & & & \\
\hline $\begin{array}{l}\text { Both primary tumor and its thrombus } \\
\text { Only tumor thrombus }\end{array}$ & $1.1(0.5-2.6)$ & 0.8 & & \\
\hline
\end{tabular}

V30Gy was evaluated in 48 patients whose data was not lost. The median volume of V30Gy of the normal liver was 14\% (range of $1-55 \%$ ). Median EQD2 and BED irradiated to the normal liver were 9 Gy (range of 1-26) and 14 Gy (range of 143), respectively.

\section{Discussion}

We retrospectively evaluated the treatment results of RT with or without chemotherapy for 64 patients with HCC that invaded PV, HV, IVC, or BD.

Although there are various treatment modalities for advanced HCC, including sorafenib, regorafenib, lenvatinib, surgery, TACE, HAIC, and RT, no standard therapeutic options are as of yet established.

In particular, the selection of treatment for $\mathrm{HCC}$ with a macroscopic vascular invasion (MVI) that causes poor prognosis is still controversial. Sorafenib and regorafenib have demonstrated significant benefits for OS and safety in the analysis of randomized phase III trials $[5,6,14,15]$. The trials showed that the median OS for patients with advanced HCC, a well-preserved liver function, and undergoing treatment with sorafenib was 7-11 months, and the ORR was only $2-3 \%$. Although the disease control rate, that was defined as the percentage of patients with $\mathrm{CR}, \mathrm{PR}$, or SD based on radiologic review, was $43-53 \%$. In a nationwide survey of advanced HCC in Japan it was shown that HAIC can have a significant positive impact on OS, the median OS was 14 months in patients with advanced $\mathrm{HCC}$ and 8.8 months longer than the control group [8]. Analysis in a non-randomized prospective study showed that TACE can be an effective treatment for PVTT, and the one-year OS rate was $18 \%$ and the ORR was $20 \%$ [7]. Although the multicenter cohort studies of surgery for PVTT showed that the five-year OS was $10-39 \%$ and median survival time was $11-21$ months [16-18], surgery is generally not feasible in patients with advanced HCC due to the spreading of multiple intrahepatic tumors or insufficient function of the remaining liver. As described above, sorafenib, regorafenib, TACE and HAIC have been the standard of treatment for advanced and inoperable HCC on the basis of large trials in Japan. However, as shown in the table 8, the patient backgrounds in these studies were heterogenous and only subgroup analyses have been performed to assess patients with MVI.

On the other hand, previous studies have suggested the potential therapeutic role of RT in patients with MVI of HCC, and the OS rates in those studies were better than any other studies with other treatment modalities. A large multicenter study has already assessed the efficacy of RT [19], and the one-year OS of patients with PVTT was found to be $43 \%$ and the ORR was 52\%. Moreover, the PVTT responders had a better median survival time than the non-responders (14 months versus 6 months, $\mathrm{p}<0.05)$. A randomized clinical trial have also revealed the efficacy of the combined treatment of TACE and RT [20]. The median OS of patients with MVI was found to be 14 months and significantly longer than sorafenib 
( 14 months versus 11 months, $\mathrm{p}=0.04$ ). In our study, the OS rate was similar to that of the multicenter study, and the OS rate of responders was also significantly better than that of non-responders $(75 \%$ versus $35 \%, p=0.009)$. The objective response must be an important endpoint, because reducing the tumor thrombosis size can delay intravascular tumor growth and the deterioration of liver function, by preserving adequate vascular flow, as well as by facilitating subsequent treatment of the primary tumor.

Table 8. Comparison of clinical results for advanced HCC among treatment modalities.

\begin{tabular}{|c|c|c|c|c|c|c|}
\hline & $\begin{array}{l}\text { No. of all } \\
\text { the Pts }\end{array}$ & $\begin{array}{l}\text { No. of Pts with } \\
\text { macrovascular invasion }\end{array}$ & $\begin{array}{l}\text { Objective Responses } \\
\text { rate }(\%)\end{array}$ & $\begin{array}{l}\text { Response of whole tumor or } \\
\text { only tumor thrumbus }\end{array}$ & $\begin{array}{l}\text { 1-year OS } \\
(\%)\end{array}$ & $\begin{array}{l}\text { Median } \\
\text { OS }\end{array}$ \\
\hline \multicolumn{7}{|l|}{ Regorafenib [5] } \\
\hline & 379 & 110 & 11 & Whole & NA & 11 \\
\hline & 299 & 108 & 2 & Whole & 44 & 11 \\
\hline & 150 & 54 & 5 & Whole & NA & 7 \\
\hline TACE [7] & 84 & 84 & 20 & Whole & 18 & 5 \\
\hline \multicolumn{7}{|l|}{ HAIC [8] } \\
\hline \multirow{2}{*}{ RT [11] } & 476 & NA & 40 & Whole & NA & 14 \\
\hline & 985 & 985 & 52 & Only tumor thrombus & 43 & 10 \\
\hline
\end{tabular}

HCC, Hepatocellular carcinoma; Pts, Patients; OS, Overall survival; RT, Radiation therapy; HAIC, Hepatic arterial infusion chemotherapy; TACE, Transarterial chemoembolization.

We analyzed the prognostic factors relating to the ORR, which was evaluated using dynamic imaging studies according to the modified criteria, and a prescribed dose was a unique significant factor (Odds ratio 3.1, 95\% CI [0.95, 10.4], $\mathrm{p}=0.04$ ) in the univariate analysis. This finding was consistent with the results of previous studies that recommended a prescribed dose higher than 45-50 in EQD2 [2, 19-23]. Although some studies emphasized the impact of the local response in relation to the OS, few studies have suggested factors relating to the ORR. The Child-Pugh class, T stage, cause of hepatitis, tumor size, site of tumor thrombosis (PV, $\mathrm{HV}$, IVC, or BD), combined chemotherapy including sorafenib, HAIC, or TACE, none of them were found to be related with ORR in our study.

The OS of patients with EOCG PS 0-1, Child-Pugh class A, T2 stage, a primary tumor size less than $4 \mathrm{~cm}$, a tumor thrombus length of less than $3.2 \mathrm{~cm}$, more than $48.75 \mathrm{~Gy}$ of EQD2, combined chemotherapy, the response of the tumor thrombus, or both primary tumor and tumor thrombus irradiated was significantly better than that of other patients, in the univariate analysis. The Child-Pugh class A was extracted as a significant prognostic factor among all the aforementioned factors, in the multivariate analysis.

However, in the study of HCC with MVI, survival rates and prognostic factor analysis tends to be difficult to assess due to the competing risk bias and the selection bias. Many patients with HCC were originally suffering from chronic liver damage due to a virus or heavy alcohol consumption. Treatment could worsen liver damage and shorten the prognosis. Furthermore, the method of treatment before and after radiotherapy varies according to the patient's background and the primary tumor. To resolve the competing risk bias and the selection bias, CIF were used to evaluate the risk factors.

In our study, ROC curve analysis was used to calculate a threshold value for the length of tumor thrombus $\mathrm{s}$ in relation to response, and that was found to be $3.2 \mathrm{~cm}$. To our knowledge, this study for the first time used a three-dimensional image analysis system to measure the thrombus and there has been no study that has demonstrated the significant correlation between the length of tumor thrombus and OS, ORR, or CIF. Previous studies would have bias for measurement of the tumor thrombus because it's difficult to evaluate long and curved tumor thrombus. It was interesting to note that the length of tumor thrombus was only a unique factor related with CIF for the progression of the intrahepatic tumor, in the univariate and multivariate analysis, although the local response was significantly related with OS and the significant prognostic factor of local response was the RT dose (EQD2).

Moreover, a small difference of 6.4 Gy in EQD2 unexpectedly resulted in a significant impact on response. This might be because short tumor thrombi tended to be irradiated in 48.75 Gy of EQD2 and long tumor thrombi in 42.35 Gy in order to minimize the risk of the liver damage.

Yoon et al. [1] treated PVTT with a combination of TACE and RT and reported that the involvement of main or bilateral $\mathrm{PV}$, a higher level of $\alpha$-fetoprotein (AFP), and advanced modified UICC stage were independent predictors of decreased OS. Pao et al. [2] treated IVCTT with RT and reported that the median OS was significantly longer for patients with Child-Pugh class A, without LN metastasis, and without lung metastasis. The results of these previous studies have shown that the OS was influenced by factors that affected liver functions and systemic dissemination. The size of the tumor thrombus must influence the liver function because of the blood supply, and the liver dysfunction causes portal hypertension resulting in the rupture of collateral vessels, ascites, and hepatic encephalopathy. Even if CR or PR is not achieved for a patient with a small tumor thrombus, the maintaining SD must lead to a long-term prognosis, and small 
field irradiation reduces the risk of radiation-induced liver disease (RILD).

The general guideline was that the fraction of the normal liver treated with more than $50 \%$ of prescribed dose should be less than $50 \%$ of the normal liver volume (V50\% $<50 \%$ ), and the volume of the normal liver that was damaged by irradiation was defined as the fraction volume of normal liver that received more than 30 Gy (V30Gy), with no more than $30 \%$ of the normal liver exposed to more than 30 Gy $(\mathrm{V} 30 \mathrm{~Gy}<30 \%)$ [24]. In our study, the correlation between RILD and a dose-volume histogram was not acknowledged. This might be because we used the system of respiratory-gated irradiation to reduce the volume of the liver that was irradiated.

On the other hand, CIF for the deterioration of the Child-Pugh class suggested that acute liver damage was a unique predictor of the deterioration of the Child-Pugh class. This seems logical in the sense that chronic and irreversible liver damage was triggered by the acute liver damage event.

Recently, some studies have reported the efficacy of SRT or proton beam irradiation for HCC with MVI [22, 23, 25-27]. Focal irradiation using such a high-tech RT may be able to help change the strategy for this disease with poor prognosis.

In our study, neither the $\mathrm{N}$ nor $\mathrm{M}$ factor was significantly related to OS. This might be because systemic treatment controlled those metastases and their prognosis was not different from the patients without $\mathrm{N}$ or $\mathrm{M}$ factor. Prospective studies are needed to evaluate the efficacy of combination therapy between the local treatment of RT and systemic treatment of sorafenib for the treatment of advanced HCC with $\mathrm{N}$ and/or $\mathrm{M}$ factors. At least in CIF of the deterioration of the Child-Pugh class and the progression of the intra/extrahepatic tumor, combined chemotherapy with RT was not risk-related.

Limitation of this study is inhomogeneity of patients' characteristics, as the number of patients with HCC including MVI is relatively small even in multi-center study, and we excluded the patients who didn't undergo follow-up dynamic imaging studies after completion of RT to evaluate the ORR. Furthermore, this was a multi-center retrospective study, and because the dose-volume histograms were not calculated in 16 of all the patients due to a mechanical failure and the duration of follow-up was limited, it was impossible to fully evaluate any adverse effects on the liver function. Nevertheless, we consider that the ORR and CIF could be accurately assessed in this study without bias.

\section{Conclusion}

RT was a useful treatment for HCC with MVI. The local response of tumor thrombosis was significantly related to OS, and the RT dose was a significant prognostic factor for ORR. On the other hand, the length of the tumor thrombi significantly impacted the CIF for the progression of the intrahepatic tumor, in the multivariate analysis. The results suggest that high doses should be delivered to the tumor thrombi, but long tumor thrombi are difficult to control locally. The length of tumor thrombus would be a predictor of intrahepatic tumor progression and accurate measurement of tumor thrombus length is important for predicting prognosis.

\section{References}

[1] Yoon SM, Lim YS, Won HJ, et al. Radiotherapy plus transarterial chemoembolization for hepatocellular carcinoma invading the portal vein: long-term patient outcomes. Int $\mathrm{J}$ Radiat Oncol Biol Phys 2012; 82: 2004-11.

[2] Pao TH, Hsueh WT, Chang WL, et al. Radiotherapy for inferior vena cava tumor thrombus in patients with hepatocellular carcinoma. BMC Cancer (2019) 19: 560.

[3] Nakazawa T, Shibuya A, Okuwaki Y, et al. Overall survival in response to sorafenib versus radiotherapy in unresectable hepatocellular carcinoma with major portal vein tumor thrombosis: propensity score analysis BMC Gastroenterology 2014, 14: 84 .

[4] Kudo M, Matsui O, Izumi N, et al. Japanese Society of Hepatology Consensus-Based Clinical Practice Guidelines for the Management of Hepatocellular Carcinoma: 2014 Update by the Liver Cancer Study Group of Japan. Liver Cancer 2014; 3: $458-468$.

[5] Bruix J, Qin S, Merie P, et al. Regorafenib for patients with hepatocellular carcinoma who progressed on sorafenib treatment (RESORCE): a randomised, double-blind, placebo-controlled, phase 3 trial. Lancet 2017; 389: 56-66.

[6] Llovet JM, Ricci S, Mazzaferro V, et al. Sorafenib in advanced hepatocellular carcinoma. N. Engl. J. Med 2008; 359, 378-390.

[7] Luo J, Guo RP, Lai EC, et al. Transarterial Chemoembolization for Unresectable Hepatocellular Carcinoma with Portal Vein Tumor Thrombosis: A Prospective Comparative Study. Ann Surg Oncol (2011) 18: 413-420.

[8] Nouso K, Miyahara K, Uchida D, et al. Effect of hepatic arterial infusion chemotherapy of 5-fluorouracil and cisplatin for advanced hepatocellular carcinoma in the Nationwide Survey of Primary Liver Cancer in Japan. British Journal of Cancer (2013) 109, 1904-1907.

[9] Wang K, GuoWX, Chen MS, et al. Multimodality Treatment for Hepatocellular Carcinoma With Portal Vein Tumor Thrombus. Medicine Volume 95, Number 11, March 2016 1-10.

[10] Bai H, Gao P, Gao H, et al. Improvement of Survival Rate for Patients with Hepatocellular Carcinoma Using Transarterial Chemoembolization in Combination with Three-Dimensional Conformal Radiation Therapy: A Meta-Analysis. Med Sci Monit, 2016; 22: 1773-1781.

[11] Kondo Y, Kimura O, Kogure T, et al. Radiation Therapy Is a Reasonable Option for Improving the Prognosis in Hepatocellular Carcinoma. Tohoku J. Exp. Med., 2015, 237, 249-257.

[12] Meng MB, Cui YL, Lu Y, et al. Transcatheter arterial chemoembolization in combination with radiotherapy for unresectable hepatocellular carcinoma: A systematic review and meta-analysis. Radiotherapy and Oncology 92 (2009) 184-194.

[13] Zou LQ, Zhang BL, Chang Q, et al. 3D conformal radiotherapy combined with transcatheter arterial chemoembolization for hepatocellular carcinoma. World J Gastroenterol 2014 December 7; 20 (45): 17227-17234. 
[14] Cheng AL, Kang YK, Chen Z, et al. Efficacy and safety of sorafenib in patients in the Asia-Pacific region with advanced hepatocellular carcinoma: a phase III randomised, double-blind, placebo-controlled trial. Lancet Oncol 2009; 10: 25-34.

[15] Chen AL, Finn RS, Qin S, et al. Phase III trial of lenvatinib (LEN) vs sorafenib (SOR) in firstline treatment of patients (pts) with unresectable hepatocellular carcinoma (uHCC). J Clin Oncol 2017; 35: abstract 4001.

[16] Kokudo T, Hasegawa K, Matsuyama Y, et al. Liver Resection for Hepatocellular Carcinoma Associated With Hepatic Vein Invasion: A Japanese Nationwide Survey. HEPATOLOGY, VOL. 66, NO. 2, 2017 510-517.

[17] Pawlik T, Poon R, Abdalla E, et al. Hepatectomy for hepatocellular carcinoma with major portal or hepatic vein invasion: Results of a multicenter study. Surgery. 2005; 137: 403-10.

[18] Torzilli G, Belghiti J, Kokudo N, et al. A Snapshot of the Effective Indications and Results of Surgery for Hepatocellular Carcinoma in Tertiary Referral Centers: Is It Adherent to the EASL/AASLD Recommendations? Annals of Surgery. Volume 257, Number 5, May 2013.

[19] Im JH, Yoon SM, Park HC, et al. Radiotherapeutic strategies for hepatocellular carcinoma with portal vein tumour thrombosis in a hepatitis B endemic area. Liver Int 2017; 37: 90-100.

[20] Yoon SM, Ryoo BY, Lee SJ, et al. Efficacy and Safety of Transarterial Chemoembolization Plus External Beam Radiotherapy vs Sorafenib in Hepatocellular Carcinoma With Macroscopic Vascular Invasion. A Randomized Clinical Trial. JAMA Oncol. 2018; 4 (5): 661-669.
[21] Choi Y, Kim JW, Cha H, et al. Overall response of both intrahepatic tumor and portal vein tumor thrombosis is a good prognostic factor for hepatocellular carcinoma patients receiving concurrent chemoradiotherapy. Journal of Radiation Research, 2014, 55, 113-120.

[22] Matsuo Y, Yoshida K, Nishimura $\mathrm{H}$, et al. Efficacy of stereotactic body radiotherapy for hepatocellular carcinoma with portal vein tumor thrombosis/inferior vena cava tumor thrombosis: evaluation by comparison with conventional three-dimensional conformal radiotherapy. Journal of Radiation Research, Vol. 57, No. 5, 2016, pp. 512-523.

[23] Xi M, Zhang L, Zhao L, et al. Effectiveness of Stereotactic Body Radiotherapy for Hepatocellular Carcinoma with Portal Vein and/or Inferior Vena Cava Tumor Thrombosis. PLoS ONE, 2013, 8 (5): e63864.

[24] Marks LB, Yorke ED, Jackson A, Haken RKT, Constine LS, Eisbruch A, et al. Use of normal tissue complication probability models in the clinic. Int J Radiat Oncol Biol Phys 76 (3 Suppl): S10-19, 2010.

[25] Sugahara S, Nakayama H, Fukuda K, et al. Proton-Beam Therapy for Hepatocellular Carcinoma Associated with Portal Vein Tumor Thrombosis. Strahlenther Onkol 2009; 185: 7828.

[26] Lee SU, Park JW, Kim TH, et al. Effectiveness and safety of proton beam therapy for advanced hepatocellular carcinoma with portal vein tumor thrombosis. Strahlenther Onkol, 2014, 190, 806-814.

[27] Sekino Y, Okumura T, Fukumitsu N, et al. Proton beam therapy for hepatocellular carcinoma associated with inferior vena cava tumor thrombus. Journal of Cancer Research and Clinical Oncology (2020) 146: 711-720. 